Pacific Journal of Mathematics

THE ABEL-JACOBI ISOMORPHISM FOR THE SEXTIC 


\section{THE ABEL-JACOBI ISOMORPHISM FOR THE SEXTIC DOUBLE SOLID}

\section{Giuseppe Ceresa And Alessandro Verra}

Let $X$ be a double cover of $\mathbf{P}^{3}$ branched along a sextic surface $S$. Using a method of Clemens and Letizia, in this paper we show that, for general $X$, the Abel-Jacobi map associated to the surface $F$ of curves contained in $X$ which are preimages of conics "totally tangent" to $S$, induces an isomorphism between the Albanese variety of $F$ and the intermediate jacobian of $X$.

0. Let $f: X \rightarrow \mathbf{P}^{3}$ be a double cover of $\mathbf{P}^{3}$ branched over a smooth sextic surface $S$. Let $F(X)$ be the Fano variety parametrizing "conics" contained in $X$; i.e., rational curves $C^{\prime} \subseteq X$ such that $C=f_{*}\left(C^{\prime}\right)$ is a reduced degree 2 plane curve, and $f^{*} f_{*}\left(C^{\prime}\right)=C^{\prime}+i_{*}\left(C^{\prime}\right)$, where $i$ is the involution associated to $f$. For generic $S, F(X)$ is an unbranched double covering of the variety $F(S)$ parametrizing conics which are totally tangent to $S$; i.e., conics in $\mathbf{P}^{3}$ having everywhere even order contact with the branch locus $S$.

After showing that, for generic $X, F(X)$ is a smooth irreducible surface, in $\S 3$ of this paper we prove that the Abel-Jacobi map

$$
a: \operatorname{Alb}(F(X)) \rightarrow J(X)
$$

between the Albanese variety of $F(X)$ and the intermediate Jacobian of $X$, is, for generic $X$, an isomorphism. The proof is based on Clemens' method, as described in [3], [5], [7]; in particular see [10] p. 478. As for the motivation for studying the map $a$, and all the necessary definitions, the reader is referred to the introduction of most of the papers cited in the references, and in particular to the survey article [4].

In $\$ 1$ we study the variety $F(S)$. In particular, using elementary deformation theory, we show that, for generic $S, F(S)$ is a smooth irreducible surface. In addition, in the space $\mathbf{P}^{83}$ of all sextic surfaces, we describe the codimension-1 locus parametrizing singular $F(S)$. More precisely, we show that this subset is the union of two hypersurfaces, $A^{\prime}$, and $B^{\prime} ; A^{\prime}$ corresponding to special sextics (see Def. 1.2), and $B^{\prime}$ parametrizing singular sextics. For $S=$ generic point of $A^{\prime}, F(S)$ will only have isolated singularities. In $\$ 2$ we discuss the case of a sextic surface with one ordinary node. In this situation, for general $S, F(S)$ is 
singular along the curve $F(S)_{0}$ parametrizing totally tangent conics passing smoothly through the node of $S$ : here we show that $F(S)_{0}$ is a smooth irreducible curve. Also, in this Section we construct the normalization $n: \widetilde{F(S)} \rightarrow F(S)$ of $F(S)$, and in $\widehat{F(S)}$ we identify the preimage $\widehat{F(S)_{0}}$ of $F(S)_{0}$.

Finally, in order to show that $a$ is an isomorphism, it is enough to prove that, modulo torsion, the cylinder map $\gamma: H_{1}(F(X), Z) \rightarrow H_{3}(X, Z)$ is an isomorphism. Clemens' method reduces this assertion to the verification of a series of facts concerning the rational family of Fano varieties $\left\{F\left(X_{t}\right)\right\}\left(t \in \mathbf{P}^{1}\right)$, associated to a general family $\left\{X_{t}\right\}\left(t \in \mathbf{P}^{1}\right)$ of sextic double solids (see Prop. 3.2). This is done in $\S 3$, using the results from the previous two sections.

We end this introduction recalling a few general facts concerning (sextic) double solids. Let $F=0$ be the equation of the sextic surface $S$. Then, the double solid $X$ is nonsingular if, and only if, $S$ is nonsingular. Denoting with $E$ the line bundle $\mathcal{O}_{\mathbf{p}^{3}}(3)$, then $f$ factors naturally as a closed embedding and a bundle projection:

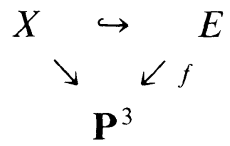

In this way $X$ is identified with the zero scheme in $E$ of $T^{2}-F \in$ $H^{0}\left(E, f^{*} \mathcal{O}_{\mathbf{P}^{3}}(3)\right)$, where $T=$ fibre coordinate; and $S$ is defined inside $X$ by the equation $T=0$ [compare 11,pp.7-9]. So, setting $\mathcal{O}_{E}(n)=f^{*} \mathcal{O}_{\mathbf{p}^{3}}(n)$, we obtain the following identification:

$$
\mathcal{O}_{E}(X) \simeq \mathcal{O}_{E}(6), \quad \mathcal{O}_{X}(S) \simeq \mathcal{O}_{X}(3)
$$

and, for the sheaves of relative differentials:

$$
\Omega_{E / \mathbf{P}^{3}}^{1} \simeq \mathcal{O}_{E}(-3), \quad \Omega_{X / \mathbf{P}^{3}}^{1} \simeq \mathcal{O}_{S}(-3) .
$$

Finally, setting $H=f^{-1}\left(\mathbf{P}^{2}\right)$, where $\mathbf{P}^{2} \subseteq \mathbf{P}^{3}$ is a generic plane, from the exact sequence

$$
0 \rightarrow f^{*} \Omega_{\mathbf{P}^{3}}^{1} \rightarrow \Omega_{X}^{1} \rightarrow \Omega_{X / \mathbf{P}^{3}}^{1} \rightarrow 0
$$

we get $K_{X}=c_{1}\left(\Omega_{X}^{1}\right)=-4 H+3 H=-H$.

This shows that $X$ is a (hyperelliptic) Fano three-fold of index 1, and of the first species, since, by a Lefschetz type theorem and the fact that $X$ can be realized alternatively as a smooth hypersurface of degree 6 in a weighted projective space $P\left(x_{0}, \ldots, x_{3}, T\right)$ where $\operatorname{deg}\left(x_{l}\right)=1$ for $i=$ $0, \ldots, 3$, and $\operatorname{deg}(T)=3, \operatorname{Pic}(X)=Z[9]$.

Throughout this paper we work over the field of the complex numbers. 
1. Let $\mathscr{Z}_{d}$ be the variety parametrizing the pairs $(C, H)$ where $H \subseteq \mathbf{P}^{3}$ is a plane and $C \subseteq H$ is a degree $d \geq 1$ curve. Since $\mathscr{Z}_{d}$ is a $\mathbf{P}^{N}$-bundle over the dual space $\left(\mathbf{P}^{3}\right)^{*}$, where $N=\left({ }^{d+2}\right)-1, \mathscr{Z}_{d}$ is a non singular irreducible variety of dimension $N+3$.

In $\mathbf{P}^{3}\left(x_{0}: x_{1}: x_{2}: x_{3}\right)$ consider the (general) sextic surface $S$ defined by the equation:

$$
h_{0} x_{0}^{6}+h_{1} x_{0}^{5}+\cdots+h_{5} x_{0}+g_{2} g_{4}+g_{3}^{2}=0
$$

where $h_{i}, i=0, \ldots, 5$, resp. $g_{j}, j=2,3,4$, are generic forms in $x_{1}, x_{2}$, $x_{3}$ of degrees $i$, resp. $j$. Then, on the plane $H_{0}=\left\{x_{0}=0\right\}$ the equation $g_{2}=0$ gives a conic $C_{0}$ which we define as totally tangent to $S$. By Noether's theorem $G_{0}=\left\{g_{2} g_{4}+g_{3}^{2}=0\right\}$ is the equation of a general plane sextic with a totally tangent conic $C_{0}$; moreover equation (1.1) defines a general sextic surface, as one can also verify by counting parameters.

DeFINITION 1.2. We say that a sextic surface is special if it admits a totally tangent conic such that, with the above choice of coordinates, $h_{5} \in\left(g_{2}, g_{3}, g_{4}\right)$.

Assume from now on that the sextic $S$ does not contain a plane. Set

$$
\begin{aligned}
F(S) & =\left\{(C, H) \in \mathscr{Z}_{2}: C \text { is totally tangent to } S\right\} \\
& =\left\{((C, H),(G, H)) \in \mathscr{Z}_{2} \times \mathscr{Z}_{6}: C\right. \text { is totally tangent }
\end{aligned}
$$

to the plane sextic $G=H \cdot S\}$

If $\mathscr{D} \subseteq \mathscr{Z}_{2} \times \mathscr{Z}_{6}$ is the incidence divisor,

$$
\mathscr{D}=\{((C, H),(G, H)): C \text { is totally tangent to } G\}
$$

and $p_{1}, p_{2}$ are the natural projections, then, as a scheme $F(S)=\mathscr{D}$. $\left(\mathscr{Z}_{2} \times \mathscr{S}\right)$, where $\mathscr{S} \subseteq \mathscr{Z}_{6}$ is the variety parametrizing the plane sections of $S$.

LEMma 1.4. $\mathscr{D}$ is a 29 dimensional irreducible variety.

Proof. We will show that all the fibres of $p_{1}$ are irreducible and of the same dimension. In fact, if $(C, H) \in \mathscr{Z}_{2}$, then $p_{1}^{-1}(C, H)=$ sextic curves in $H=\mathbf{P}^{2}\left(x_{1}: x_{2}: x_{3}\right)$ totally tangent to $C$. Assuming $C=\left\{g_{2}=0\right\}$ and choosing coordinates as in (1.3)

$$
G \in p_{1}^{-1}(C, H) \quad \text { iff } \quad G=\left\{g_{2} g_{4}+g_{3}^{2}=0\right\}
$$


where, if $R=\mathbf{C}\left[x_{1}, x_{2}, x_{3}\right]=\oplus R_{d}$, then $g_{k} \in R_{k}$. If $\left(x_{1} g_{2}, x_{2} g_{2}\right.$, $\left.x_{3} g_{2}, W_{0}, \ldots, W_{6}\right)$ is a basis for $R_{3}$, let $W=$ vector subspace generated by $W_{0}, \ldots, W_{6}$, then, the squaring map between $W$ and $R_{6}$ induces the Veronese embedding $v: \mathbf{P}(W) \rightarrow \mathbf{P}\left(R_{6}\right)$. In $\mathbf{P}\left(R_{6}\right)$ let $N$ be the 14-dimensional linear subspace of curves containing $C$ as a component. Then we have

$(+) \quad$ iff $G$ belongs to the pencil $a\left(g_{2} g_{4}\right)+b g_{3}^{2}=0$

iff $G$ lies on the projecting cone $V$ of $\operatorname{Im}(v)$ from $N$.

Finally, since $\operatorname{Im}(v) \cap N=\varnothing, V$ is 21 dimensional and irreducible.

REMARKs. (a) One can also check that

$$
\operatorname{Sing}(\mathscr{D})=\{(G, H) \in \mathscr{D}: G \text { contains a conic }\}
$$

(b) Notice that $\operatorname{Sing}(V)=N$; for, the intersection of $N$ and $\operatorname{Sec}(\operatorname{Im}(v))$ is empty. In fact, it is known that for the Veronese embedding $T$ of $\mathbf{P}^{n}$, $\operatorname{dim}(\operatorname{Sec}(T))=2 n$ instead of $2 n+1$; moreover a pencil $\left\{a g_{3}^{2}+b f_{3}^{2}=0\right\}$ $\left(g_{3}, f_{3} \in W\right)$ cannot intersect the linear system $N$.

(c) From the previous remark we have that $\mathscr{Z}_{2} \times \mathscr{S}$ and $\mathscr{D}$ are smooth along their intersection provided $S$ does not contain a conic. Since $\operatorname{dim}\left(\mathscr{Z}_{2} \times \mathscr{S}\right)+\operatorname{dim}(\mathscr{D})=2+\operatorname{dim}\left(\mathscr{Z}_{2} \times \mathscr{Z}_{6}\right), F(S)$ is smooth and 2-dimensional at $P$ iff $\mathscr{Z}_{2} \times \mathscr{S}$ and $\mathscr{D}$ intersect transversally at $P$.

Using elementary deformation theory, we would like to compute the dimension of the tangent space to $F(S)$ in $0=\left(\left(C_{0}, H_{0}\right),\left(G_{0}, H_{0}\right)\right)$.

The local deformation of 0 in $\mathscr{Z}_{2} \times \mathscr{Z}_{6}$ is given by:

$$
\begin{aligned}
& \left\{\begin{array}{l}
g_{2}+\sum_{i=1}^{5} u_{i}^{\prime} U_{i}=0 \\
x_{0}=t_{1}^{\prime} x_{1}+t_{2}^{\prime} x_{2}+t_{3}^{\prime} x_{3}
\end{array}\right. \\
& \left\{\begin{array}{l}
\left(g_{2}+\sum_{i=1}^{5} u_{l} U_{l}\right)\left(g_{4}+\sum_{j=1}^{14} v_{j} V_{j}\right) \\
\quad+(1+s)\left(g_{3}+\sum_{k=1}^{6} w_{k} W_{k}\right)^{2}+r h=0 \\
x_{0}=t_{1} x_{1}+t_{2} x_{2}+t_{3} x_{3}
\end{array}\right.
\end{aligned}
$$

where $\left(g_{2}, U_{1}, \ldots, U_{5}\right),\left(g_{4}, V_{1}, \ldots, V_{14}\right),\left(x_{1} g_{2}, x_{2} g_{2}, x_{3} g_{2}, g_{3}, W_{1}, \ldots, W_{6}\right)$ are bases of the vector spaces $R_{2}, R_{4}$, and $R_{3}$ respectively, $h$ is a generic sextic plane curve, in particular not possessing totally tangent conics, and $\left(t_{h}, u_{i}, v_{j}, w_{k}, r, s, t_{h}^{\prime}, u_{i}^{\prime}\right) \in \mathbf{A}^{38}=$ parameter space of the deformation. 
Now, to get linear deformations, we multiply by $\varepsilon$ the parameters and compute modulo $\varepsilon^{2}$. Then, from (1.5a) and (1.5b) we obtain the following linear system of curves:

$$
\left\{\begin{array}{l}
g_{2} g_{4}+g_{3}^{2}+g_{2} \sum_{i=1}^{14} v_{i} V_{i}+g_{4} \sum_{j=1}^{5} u_{j} U_{J}+s g_{3}^{2} \\
\quad+2 g_{3} \sum_{k=1}^{6} w_{i} W_{l}+r h=0 \\
x_{0}=\sum_{h=1}^{3} t_{h} x_{h}
\end{array}\right.
$$

The local deformation of 0 in $\mathscr{D}$ is obtained from (1.5a) and (1.5b) setting

$$
r=0, \quad t_{h}=t_{h}^{\prime}, h=1,2,3, \quad \text { and } \quad u_{l}=u_{i}^{\prime}, i=1, \ldots, 5
$$

Together (1.6) and (1.7) describe the tangent space $T_{\mathscr{D}, 0}$.

On the other hand, in $\mathscr{Z}_{2} \times \mathscr{S}$ the local deformation of 0 is given by (1.5a) together with the following equations:

$$
\left\{\begin{array}{l}
h_{0}\left(\sum_{h=1}^{3} t_{h}^{\prime \prime} x_{h}\right)^{6}+\cdots+h_{5}\left(\sum_{h=1}^{3} t_{h}^{\prime \prime} x_{h}\right)+g_{2} g_{4}+g_{3}^{2}=0 \\
x_{0}=\sum_{h=1}^{3} t_{h}^{\prime \prime} x_{h}
\end{array}\right.
$$

Writing the parameters of (1.5b) as polynomial functions of $\left(t_{1}^{\prime \prime}, t_{2}^{\prime \prime}, t_{3}^{\prime \prime}\right)$, with the identifications $t_{h}=t_{h}^{\prime \prime} h=1,2,3$, the family of curves (1.8) becomes, in a natural way, a subfamily of (1.5b). From equations (1.5) and (1.8) we see that $T_{\mathscr{Z}_{2} \times \mathscr{S}, 0} \subseteq T_{\mathscr{Z}_{2} \times \mathscr{Z}_{6}, 0}$ can be identified with the linear system of plane sextics:

$$
\left\{\begin{array}{l}
h_{5}\left(\sum_{h=1}^{3} x_{h}\right)+g_{2} g_{4}+g_{3}^{2}=0 \\
x_{0}=\sum_{h=1}^{3} t_{h} x_{h}
\end{array}\right.
$$

Since in $T_{\mathscr{Q}_{2} \times \mathscr{Q}_{6}, 0}, T_{F(S), 0}=T_{\mathscr{Q}_{2} \times \mathscr{S}, 0} \cap T_{\mathscr{D}, 0}$ we have the following:

Proposition 1.10. $\operatorname{Dim}\left(T_{F(S), 0}\right) \geq 3$ if, and only if, either

(a) $S$ is special, or

(b) the variety $V\left(h_{5}, g_{2}, g_{3}, g_{4}\right)$ defined by the ideal $\left(h_{5}, g_{2}, g_{3}, g_{4}\right)$ is a non-empty set. 
Proof. First, notice that $\operatorname{dim}\left(T_{F(S), 0}\right) \geq 3$ iff $T_{\mathscr{2} 2 \times \mathscr{S}, 0} \subseteq T_{\mathscr{D}, 0}$. Then, since we are inside the linear subspace of $\mathbf{A}^{38}$ defined by $u_{l}=u_{i}^{\prime}, i=$ $1, \ldots, 5, t_{j}=t_{\jmath}^{\prime}, j=1,2,3$; this last condition is equivalent to the fact that the family of plane sextics (1.9) is contained in the family (1.6) where $r=0$. Thus, $h_{5}\left(\sum_{h=1}^{3} t_{h} x_{h}\right) \subseteq\left(g_{2}, g_{3}, g_{4}\right)$. Case (a) follows from this (by Macauley's theorem [8] p. 599) when $\left\{g_{2}=g_{3}=g_{4}=0\right\}=$ empty, and (b) simply because $S$ is singular in $0 \in V\left(h_{5}, g_{2}, g_{3}, g_{4}\right), h_{4}(0)=0$ and (1.6) is the linear system of plane sextics passing through 0 .

REMARK. If $S$ has a node in 0 , then every totally tangent conic smooth in 0 satisfies condition (b) of the proposition. Notice that, passing through 0 there are finitely many tritangent lines. Any two of these tritangents give a reduced totally tangent conic singular in 0 . However, for generic $S$ with a node, these conics do not satisfy condition (b), because in this case one gets $g_{4}(0) \neq 0$, so they correspond to smooth points of $F(S)$.

Next, we want to show that, for generic $S, F(S)$ is a smooth irreducible surface.

If $\mathbf{P}^{83}$ is the projective space parametrizing all sextic surfaces in $\mathbf{P}^{3}$, consider the correspondence:

$$
\begin{array}{cc}
\mathscr{R} & \stackrel{q_{1}}{\rightarrow} \\
q_{2} \downarrow & \mathbf{P}^{83} \\
\mathscr{Z}_{2} \times \mathscr{Z}_{6} &
\end{array}
$$

where $\mathscr{R}=\{((C, H),(G, H), S): H \cdot S=G\}$.

Set $D=q_{2}^{*}(\mathscr{D})$, and let $q_{1}, i=1,2$, also denote the restriction to $D$ of the projections in (1.11).

LEMMA 1.12. $D$ is an irreducible variety.

Proof. This follows from Lemma 1.4 since all fibres of $q_{2}$ are isomorphic to $\mathbf{P}^{56}$.

Now, in $D$ consider the subset $T$ defined as

$$
T=\left\{0 \in D: \operatorname{dim}\left(T_{F(S), 0}\right) \geq 3\right\}
$$

and let $A$, resp. $B$, be the locus defined by the condition (a), resp. (b), of Proposition 1.10.

Lemma 1.13. (i) $A$ is irreducible, $\operatorname{codim}(A)=3, \operatorname{codim}(B)=2$ and $T=A \cup B$ 
(ii) $q_{1^{*}}(A)=A^{\prime}, q_{1^{*}}(B)=B^{\prime}$, where $A^{\prime}$ parametrizes the set of special sextics, and $B^{\prime}$ the set of singular sextic surfaces;

(iii) $\operatorname{codim}\left(A^{\prime} \cap B^{\prime}\right) \geq 2$.

Proof. With the same notation as in the previous lemma, set $0=$ $((C, H),(G, H)) \in \mathscr{D}$. Then

$$
q_{2}^{-1}(0)=\text { linear system }\left\{Q x_{0}+t\left(g_{2} g_{4}+g_{3}^{2}\right)=0\right\} \simeq \mathbf{P}^{56},
$$

where $Q \in H^{0}\left(\mathbf{P}^{3}, \mathcal{O}_{\mathbf{P}^{3}}(5)\right)$, and $t \in \mathbf{C}$. Also,

$$
q_{2}^{-1}(0) \cap A=\text { linear system }\left\{x_{0}^{2} P+x_{0} U+t\left(g_{2} g_{4}+g_{3}^{2}\right)=0\right\} \text {, }
$$

where $P \in H^{0}\left(\mathbf{P}^{3}, \mathcal{O}_{P^{3}}(4)\right)$. If $I_{5}=\left(g_{2}, g_{3}, g_{4}\right)$ then $U \in I_{5} \subseteq R_{5}$. Since $\operatorname{dim}\left(I_{5}\right)=18$, and $\operatorname{dim} H^{0}\left(H, \mathcal{O}_{H}(5)\right)=21$, it follows that $q_{2}^{-1}(0) \cap A \simeq$ $\mathbf{P}^{53}$, so $A$ is a codimension 3 irreducible variety. In particular, $\operatorname{codim}\left(A^{\prime}\right)$ $\geq 1$. Also, $S \in q_{2}^{-1}(0) \cap B$ iff $V=V\left(h_{5}, g_{2}, g_{3}, g_{4}\right) \neq \varnothing$. Since $\operatorname{Sing}(S)$ $\supseteq V, q_{1}(B)=B^{\prime}$. In addition, $\operatorname{codim}(B)=2$. For, on the one hand, $Y=q_{2}(B) \subseteq \mathscr{D}$ is an irreducible divisor. In fact, if $p=((C, H),(G, H))$ $\in Y \subseteq \mathscr{Z}_{2} \times \mathscr{Z}_{6}$, then $V \neq \varnothing$ implies $V(I) \neq \varnothing$; then $\operatorname{Sing}(G) \neq \varnothing$ and $C \cap \operatorname{Sing}(G) \neq \varnothing$. So,

$$
Y=\{((C, H),(G, H)) \in \mathscr{D}: \operatorname{Sing}(G) \neq \varnothing \text { and } C \cap \operatorname{Sing}(G) \neq \varnothing\} .
$$

Since $p_{1}(Y)=\mathscr{Z}_{2}$, and in $Y p_{1 / Y}^{-1}(C, H)$ is an irreducible 20-dimensional variety, we get that $Y$ is an irreducible divisor on $\mathscr{D}$. On the other hand, $q_{2}^{-1}(p) \cap B=\bigcup_{u \in \operatorname{Sing} G} \mathbf{P}_{u}^{55}$ with $\mathbf{P}_{u}^{55}=$ linear system $\left\{x_{0}^{2} P+x_{0} h_{5}+\right.$ $\left.t\left(g_{2} g_{4}+g_{3}^{2}\right)=0\right\}$, where $t \in \mathbf{C}, \quad P \in H^{0}\left(\mathbf{P}^{3}, \mathcal{O}_{\mathbf{P}^{3}}(4)\right), \quad h_{5} \in R_{5}$ and $h_{5}(u)=0$.

To see (iii), notice that $A^{\prime} \nsubseteq B^{\prime}$. For, consider the linear system $\left\{x_{0}^{2} P+x_{0} h_{5}+g_{2} g_{4}+g_{3}^{2}=0\right\}$, where $P$ varies in $H^{0}\left(P^{3}, O_{P^{3}}(4)\right), h_{5} \in$ $\left(g_{2}, g_{3}, g_{4}\right)$ and $\left\{g_{2} g_{4}+g_{3}^{2}=x_{0}=0\right\}=G$ is smooth. Then each surface of the system is special and, since the cone $\left\{g_{2} g_{4}+g_{3}^{2}=0\right\}$ is non singular along the base locus $G$, by Bertini's theorem, the generic element of the system is smooth.

Proposition 1.14. If $S \in\left(P^{83}-\left(A^{\prime} \cup B^{\prime}\right)\right)$ then $F(S)$ is a smooth irreducible surface.

Proof. By Lemma 1.13 we only need to show that $F(S)$ is connected, and this follows from the fact that $\operatorname{codim}(A \cup B) \geq 2$. For, if $D^{0}=D-$ $\operatorname{Sing}(D)$ and $q_{1}^{\prime}=u \circ c: D^{0} \rightarrow W \rightarrow P^{83}$ is the Stein factorization of the proper morphism $q_{1}^{\prime}=q_{1} \mid D^{0}$, then, $W$ is a normal variety and $u$ is finite. If there exists a fibre $F(S)$ which is not connected, then the general fibre of $u$ will also be disconnected. Let $K \subseteq \mathbf{P}^{83}$ be the branching divisor of $u$, and $K^{\prime}=\left(q_{1}^{\prime}\right)^{-1}(K)$ be the corresponding divisor in $D^{0}$. Since, if $p \in K^{\prime}$, 
the differential $d q_{1}^{\prime}$ is not surjective at $p[1, \mathrm{p} .101]$, we must have $\operatorname{dim}\left(T_{F(S), p}\right) \geq 3$, where $S=q_{1}(p)$. Hence $K^{\prime} \subseteq A \cup B$ which is impossible by Zariski's Theorem on the purity of the branch locus.

2. Throughout this section we make the following assumption.

Assumption 2.1. (i) $S=$ singular sextic surface with an ordinary node in $0(0: 0: 0: 1)$ as its only singularity;

(ii) $S$ does not contain any conic.

REMARKs. (1) Notice that a surface $S$ satisfying the above two conditions is represented by a point of $\mathbf{P}^{83}$-(closed subset of codim $\geq 2$ ).

(2) The first assumption implies that the tangent cone to $S$ in 0 has rank 3. In this situation, if $S$ is not special then $F(S)$ is singular along the locus parametrizing totally tangent conics through 0 and smooth there.

If $\sigma_{1}=$ blowing-up of $\mathscr{Z}_{2}$ along the subvariety $\mathscr{Z}_{2}^{1}$ of conics passing through 0 , and $\sigma_{2}=$ blowing-up of $\mathscr{Z}_{6}$ along the subvariety $\mathscr{Z}_{6}^{2}$ of sextics singular in 0 ; then we want to use the product

$$
n=\sigma_{1} \times \sigma_{2}
$$

to normalize $F(S)$.

In $\mathbf{P}^{3} \times \mathscr{Z}_{2}$ and $\mathbf{P}^{3} \times \mathscr{Z}_{6}$ respectively, consider the "universal curve":

$$
j_{2}: \mathscr{J}_{2} \rightarrow \mathscr{Z}_{2}, \quad j_{6}: \mathscr{J}_{6} \rightarrow \mathscr{Z}_{6}
$$

where $\mathscr{J}_{2}=\{(x ; C, H): x \in C\}, \mathscr{J}_{6}=\{(x ; G, H): x \in G\}$, and $j_{2}, j_{6}$ are the restrictions of the natural projections. Let $\sigma: \tilde{\mathbf{P}}^{3} \rightarrow \mathbf{P}^{3}$ be the blowing-up of $\mathbf{P}^{3}$ in 0 . Choosing affine coordinates:

$$
z=x_{0} / x_{3}, \quad x=x_{1} / x_{3}, \quad y=x_{2} / x_{3}
$$

in the affine space $\mathbf{A}^{3} \times \mathbf{A}^{2}=\left(x, y, z ; x^{\prime}, y^{\prime}\right), \tilde{\mathbf{P}}^{3}$ is defined by the equations $x=x^{\prime} z, y=y^{\prime} z$. In $\mathbf{A}^{3} \times \mathscr{Z}_{2} \times \mathscr{Z}_{6}$ the subvariety $\mathscr{J}_{2} \times \mathscr{J}_{6}$ is given by the affine form of equations (1.5a) and (1.5b). Now, in

$$
\begin{aligned}
\left(\mathbf{A}^{3} \times \mathbf{A}^{2}\right) & \times\left(\mathbf{A}^{30} \times \mathbf{A}^{3}\right) \\
& =\left(x, y, z ; x^{\prime}, y^{\prime}\right) \times\left(t_{h}, u_{i}, v_{j}, w_{k}, r, s ; \lambda_{0}, \lambda_{1}, \lambda_{2}\right)
\end{aligned}
$$

consider the variety $\tilde{\mathscr{J}}_{6}$ birational to $\mathscr{J}_{6}$, defined by the equations:

$$
\left\{\begin{array}{l}
Q_{00}=\lambda_{0} z^{2}, \quad Q_{10}=\lambda_{1} z, \quad Q_{01}=\lambda_{2} z \\
x=x^{\prime} z, \quad y=y^{\prime} z \\
\lambda_{0}+\lambda_{1} x^{\prime}+\lambda_{2} y^{\prime}+Q_{20}\left(x^{\prime}\right)^{2}+Q_{11} x^{\prime} y^{\prime}+Q_{02}\left(y^{\prime}\right)^{2} \\
\quad+\sum_{3 \leq i+j \leq 6}\left(x^{\prime}\right)^{i}\left(y^{\prime}\right)^{j} z^{i+j-2}=0 \\
z=z\left(t_{1} x^{\prime}+t_{2} y^{\prime}\right)+t_{3}
\end{array}\right.
$$


where $\Sigma Q_{i j} x^{i} y^{j}=0$ is the first of the equations in (1.5b), written in affine form, and $Q_{i j} \in \mathrm{C}\left[u_{i}, v_{j}, w_{k}, r, s\right]$.

Denoting with $\tilde{j}_{6}: \tilde{\mathscr{J}}_{6} \rightarrow \mathbf{A}^{30} \times \mathbf{A}^{3}$ the restriction of the natural projection, then we have $\tilde{j}_{6}\left(\tilde{J}_{6}\right)=\tilde{\mathscr{Z}}_{6}$, where $\tilde{\mathscr{Z}}_{6}$ is locally given by the equations:

$$
Q_{00}=\lambda_{0} t_{3}^{2}, \quad Q_{10}=\lambda_{1} t_{3}, \quad Q_{01}=\lambda_{2} t_{3}
$$

and the restriction

$$
\sigma_{2}: \tilde{\mathscr{Z}}_{6} \rightarrow \mathscr{Z}_{6}
$$

of the projection from $\mathbf{A}^{30} \times \mathbf{A}^{3}$ in $\mathbf{A}^{30}$ is a birational morphism. Also, the following diagram commutes:

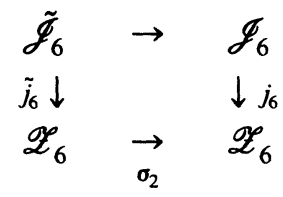

Now, if $\tilde{Q} \in \tilde{\mathscr{Z}}_{6}$ and $Q=\sigma_{2}(\tilde{Q})$, set $\tilde{G}=$ strict transform of $j_{6}^{-1}(Q)$ by $\sigma$. Except for the case in which the multiplicity of $j_{6}^{-1}(Q)$ in 0 is greater than $2,\left(\tilde{j}_{6}\right)^{-1}(\tilde{Q})$ is a curve and, either, if $Q \notin \mathscr{Z}_{6}^{2}$, this curve is biregular to $j_{6}^{-1}(Q)$, or, if $Q \in \mathscr{Z}_{6}^{2}$, is the sum $\tilde{G}+K$, where $K=$ conic in the exceptional plane $\sigma^{-1}(0)$ and $\tilde{G} \cap K=\tilde{G} \cap \sigma^{-1}(0)$.

In a similar way we construct $\tilde{\mathscr{J}}_{2}$ and $\tilde{\mathscr{Z}}_{2}$. There exists a commutative diagram:

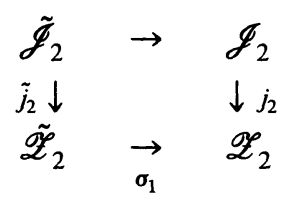

where the top arrow is the blowing-up of $\mathscr{J}_{2}$ along $j_{2}^{-1}\left(\mathscr{Z}_{2}^{1}\right)$. In $\left(\mathbf{A}^{3} \times \mathbf{A}^{2}\right)$ $\times\left(\mathbf{A}^{8} \times \mathbf{A}^{1}\right)=\left(x, y, z ; x^{\prime}, y^{\prime}\right) \times\left(u_{i}^{\prime}, t_{h}^{\prime} ; \lambda\right)$ the local equations of $\tilde{\mathscr{J}}_{2}$ are:

$$
\left\{\begin{array}{l}
R_{00}=\lambda z, \quad x=x^{\prime} z, \quad y=y^{\prime} z \\
R_{00}+R_{10} x^{\prime}+R_{01} y^{\prime}+z\left(\sum_{i+j=2} R_{i j}\left(x^{\prime}\right)^{i}\left(y^{\prime}\right)^{j}\right)=0 \\
z=z\left(t_{1}^{\prime} x^{\prime}+t_{2}^{\prime} y^{\prime}\right)+t_{3}^{\prime}
\end{array}\right.
$$

with $R_{i j} \in \mathbf{C}\left[u_{i}^{\prime}, t_{h}^{\prime}\right]$ and $\sum R_{i j} x^{i} y^{j}$ is the first equation in (1.5a). If, as in the previous case $\tilde{j}_{2}$ denotes the restriction to $\mathscr{J}_{2}$ of the canonical projection; $\tilde{\mathscr{Z}}_{2}=\tilde{j}_{2}\left(\tilde{\mathscr{J}}_{2}\right)$ is given by:

$$
R_{00}=\lambda t_{3}^{\prime} \text {. }
$$


Again, if $\tilde{P} \in \tilde{\mathscr{Z}}_{2}$ and $\sigma_{1}(\tilde{P})=P$, then $\left(\tilde{j}_{2}\right)^{-1}(\tilde{P})$ is a curve, unless $j_{2}^{-1}(P)$ is a conic singular in 0 ; and if $P \notin \mathscr{Z}_{2}^{1},\left(\tilde{j}_{2}\right)^{-1}(\tilde{P})$ is biregular to $j_{2}^{-1}(P)$, if $P \in \mathscr{Z}_{2}^{1},\left(\tilde{j}_{2}\right)^{-1}(\tilde{P})=\tilde{C}+L$. Here $\tilde{C}=$ strict transform of $j_{2}^{-1}(P)$ by $\sigma$, and $L=$ line on the exceptional plane $\sigma^{-1}(0)$ and passing through the point $\tilde{C} \cap \sigma^{-1}(0)$.

Set $E=n^{-1}\left(\mathscr{Z}_{2}^{1} \times \mathscr{Z}_{6}^{2}\right)=$ exceptional divisor of $n$. For each point $Q \in \mathscr{Z}_{2}^{1} \times \mathscr{Z}_{6}^{2}, \quad n^{-1}(Q)=\mathbf{P}^{1} \times \mathbf{P}^{3}$. If $Q=((C, H), \quad(G, H))$, with mult $_{0}(C)=1$, mult ${ }_{0}(G)=2$, then $n^{-1}(Q)$ parametrizes the pair of curves in $\sigma^{-1}(0)$

$$
(L, K) \text {, }
$$

$K$ belonging to the web of conics through $\tilde{G} \cap \sigma^{-1}(0)$, and $L$ element of the pencil of lines through $\tilde{C} \cap \sigma^{-1}(0)$. Let

$$
\overline{F(S)}=\text { strict transform of } F(S) \text { by } n,
$$

and denote also with $n$ the restriction $n \underline{F(S)}$. Given $\tilde{Q} \in \widetilde{F(S)}$, we would like to compute the tangent space to $\overline{F(S)}$ at $\tilde{Q}$.

By Proposition 1.10 and the remark following it, we have that for generic $S, \operatorname{Sing}(F(S))=\left(\mathscr{Z}_{2}^{1} \times \mathscr{Z}_{6}^{2}\right) \cdot F(S)-\left(\mathscr{Z}_{2}^{2} \times \mathscr{Z}_{6}^{2}\right) \cdot F(S)$, where $\mathscr{Z}_{2}^{2}=$ (conics singular in 0$)$. Set $E^{\prime}=\left(E-n^{-1}\left(\mathscr{Z}_{2}^{2} \times \mathscr{Z}_{6}^{2}\right)\right) \cdot \overline{F(S)}$, then $\widetilde{F(S)}-E^{\prime}$ is biregular to $F(S)-\operatorname{Sing}(F(S))$. If $\tilde{Q} \in E^{\prime}$ and $n(\tilde{Q})=Q$ $=((C, H),(G, H))$, then $Q \in \mathscr{Z}_{2}^{1} \times \mathscr{Z}_{6}^{2}$ because $G$ is a plane section through 0.

Let $\tilde{\mathscr{D}}$ and $\tilde{\mathscr{S}}$ be the strict transform of $\mathscr{D}$ by $n$ and of $\mathscr{S}$ by $\sigma_{2}$ respectively, then

$$
\overline{F(S)}=\tilde{\mathscr{D}} \cdot\left(\tilde{\mathscr{Z}}_{2} \times \tilde{\mathscr{S}}\right) \text {. }
$$

We compute now the local equation of $\tilde{\mathscr{D}}_{\text {and }} \tilde{\mathscr{Z}}_{2} \times \tilde{\mathscr{S}}$ in an open subset of $\tilde{\mathscr{Z}}_{2} \times \tilde{\mathscr{Z}}_{6}$ containing $\tilde{Q}$. Notice that (2.9) and (2.5) are the equations of $\tilde{\mathscr{Z}}_{2} \times \tilde{\mathscr{Z}}_{6}$ in $\left(\mathbf{A}^{8} \times \mathbf{A}^{1}\right) \times\left(\mathbf{A}^{30} \times \mathbf{A}^{3}\right)$.

(A) Local equations of $\tilde{\mathscr{D}}$.

The exceptional divisor of $n \mid \tilde{\mathscr{D}}$ is

$$
E \cdot \tilde{\mathscr{D}}=\{(L, K): L \text { is tangent to } K\}
$$

( $L, K$, as in (2.10)). Thus, $\tilde{\mathscr{D}}$ has equations:

$$
r=0, \quad u_{i}=u_{i}^{\prime}, i=1, \ldots, 5 ; \quad t_{h}=t_{h}^{\prime}, h=1,2,3 .
$$

plus the tangency condition:

$$
\begin{aligned}
\lambda^{2}\left(b^{2}-4 Q_{02} c\right)+ & \lambda\left(-2 b\left(\lambda_{1} R_{01}^{2}-\lambda_{2} R_{01} R_{10}\right)-4 c R_{01} \lambda_{2}\right) \\
& +(\text { const. term })=(\text { in short }) A \lambda^{2}+B \lambda+K=0
\end{aligned}
$$


between the line $\lambda+R_{10} x^{\prime}+R_{01} y^{\prime}=0$ and the conic

$$
\lambda_{0}+\lambda_{1} x^{\prime}+\lambda_{2} y^{\prime}+Q_{20}\left(x^{\prime}\right)^{2}+Q_{02}\left(y^{\prime}\right)^{2}+Q_{11} x^{\prime} y^{\prime}=0 \text {. }
$$

In (2.13)

$$
b=Q_{11}+2 Q_{02} R_{10}, \quad c=R_{01}^{2} Q_{20}-Q_{11} R_{10}+R_{01}^{2} Q_{02} .
$$

From now on we assume $R_{00}=u_{3}^{\prime}$, so that the hyper-surface

$$
u_{3}=\lambda t_{3}
$$

contains $\tilde{\mathscr{D}}$.

(B) Equations of $\tilde{\mathscr{Z}}_{2} \times \tilde{\mathscr{S}}$.

Writing the equations (1.5b) in the affine form:

$$
\sum_{i+j \leq 6} P_{i j} x^{i} y^{j}=0, \quad z=t_{1}^{\prime \prime} x+t_{2}^{\prime \prime} y+t_{3}^{\prime \prime}
$$

$P_{i j} \in \mathbf{C}\left[t_{1}^{\prime \prime}, t_{2}^{\prime \prime}, t_{3}^{\prime \prime}\right]$, in $\mathscr{Z}_{6} \mathscr{S}$ is given by:

$$
P_{i j}\left(t_{1}, t_{2}, t_{3}\right)=Q_{i j} \quad(i+j \leq 6)
$$

(after the trivial substitution $t_{h}=t_{h}^{\prime \prime}, h=1,2,3$ ). Since $S$ is singular in 0 , $t_{3}^{2}$ divides $P_{00}$, and $t_{3}$ divides $P_{01}$ and $P_{10}$, hence the equations:

$$
\tilde{P}_{00}=\lambda_{0}, \quad \tilde{P}_{01}=\lambda_{2}, \quad \tilde{P}_{10}=\lambda_{1}, \quad P_{i j}=Q_{i j} \quad i+j=2,
$$

define the strict transform of $\mathscr{S}$ in $\tilde{\mathscr{Z}}_{6}$.

If $n(\tilde{Q})=$ origin of $\mathbf{A}^{30} \times \mathbf{A}^{8}$ and $\tilde{Q} \in \tilde{\mathscr{Z}}_{2} \times \tilde{\mathscr{S}}$, then the coordinates of $\tilde{Q}$ in $\mathbf{A}^{38} \times \mathbf{A}^{4}$ are obtained setting $\lambda_{0}=h_{40}, \lambda_{1}=h_{51}, \lambda_{2}=h_{52}$, $\lambda=\lambda^{\prime}$ or $\lambda^{\prime \prime}$, where

$$
\mathscr{C}=\left\{h_{40} z^{2}+\left(h_{51} x+h_{52} y\right) z+g_{21} g_{41}+g_{31}^{2}=0\right\}
$$

is the tangent cone to $S$ in 0 , and $\lambda^{\prime}, \lambda^{\prime \prime}$ are the roots of (2.13). Set:

$$
\begin{aligned}
& F(S)_{0}=\operatorname{Sing}(F(S)) \\
& \widetilde{F(S)_{0}}=\text { inverse image of } F(S)_{0} \text { in } \widetilde{F(S)} .
\end{aligned}
$$

Then, one can compute that if the coefficients $A, B$, and $K$ of (2.13) are identically 0 , either $\operatorname{rk} \mathscr{C} \leq 1$ or $\operatorname{rk} \mathscr{C}=2$ and the conic $C$ is tangent to the intersection of the two components of $\mathscr{C}$. Hence, by our assumption on $S$, equation (2.13) defines a finite, branched double covering

$$
n \mid \widetilde{F(S)_{0}}=n_{0}: \quad \widetilde{F(S)_{0}} \rightarrow F(S)_{0} .
$$

Proposition 2.17. Let $S$ be a general sextic surface with one node, then the tangent map:

$$
d n_{Q}: T_{\widetilde{F(S)}, \tilde{Q}} \rightarrow T_{F(S), Q}
$$

is injective, unless $Q$ is a branch point for $n_{0}$. 
Proof. To simplify notation, we write $F$ for $F(S), Q=\left(Q^{\prime}, Q^{\prime \prime}\right) \in \mathscr{Z}_{2}$ $\times \mathscr{Z}_{6}$, and use similar notation for the preimages. Consider the tangent map

$$
d n_{\tilde{Q}}\left|T_{\tilde{\mathscr{L}}_{2} \times \tilde{\mathscr{S}}, \tilde{Q}}=\left(d \sigma_{1}\right)_{\tilde{Q}^{\prime}}\right| T_{\tilde{\mathscr{R}}_{2}, \tilde{Q}^{\prime}} \times\left(d \sigma_{2}\right)_{\tilde{Q}^{\prime \prime}} \mid T_{\tilde{\mathscr{S}}, \tilde{Q}^{\prime \prime}}
$$

Notice that the second map in the product is an isomorphism since $\sigma_{2}$ is biregular in $\tilde{\mathscr{S}}$. For, $\mathscr{S}$ is smooth in $\mathscr{Z}_{6}, \mathscr{Z}_{6}^{2} \cdot \mathscr{S}$ is a divisor in $\mathscr{S}$, and $\sigma_{2}$ blows-up (an ideal of) $\mathscr{Z}_{6}^{2}$. Since the fibre of $\sigma_{1}$ is $\mathbf{P}^{1}, \operatorname{dim} \operatorname{ker}\left(d \sigma_{1}\right)_{\tilde{Q}^{\prime}}=$ 1 . Thus, $d n_{\tilde{Q}}$ is injective unless $\Lambda=\operatorname{ker}\left(d \sigma_{1}\right)_{\tilde{Q}^{\prime}} \times(0) \subseteq T_{\tilde{F}, \tilde{Q}}$. Now, inside $\mathbf{A}^{38} \times \mathbf{A}^{4} \ni \tilde{Q}, \Lambda$ can be identified with the line through $\tilde{Q}$ obtained by varying the coordinate $\lambda$ (recall that $\lambda$ is a local parameter on $\mathbf{P}^{1}=$ $\left.\sigma_{1}^{-1}\left(Q^{\prime}\right)\right)$.

Since, in the above affine space, $F$ is given by the equations (2.12), (2.13), and (2.16); $\Lambda \subseteq T_{\tilde{F}, \tilde{Q}}$ (affine tangent space) iff $\Lambda$ is contained in the tangent hyperplane in $Q$ to the hypersurface (2.13). This happens iff $\lambda^{\prime}=\lambda^{\prime \prime}$; i.e. iff the two inverse images of $Q$ by $n_{0}$ coincide.

Proposition 2.18. Let $S$ be a general sextic surface with a node, then $F(S)_{0}$ is a smooth irreducible curve.

Proof. In order to prove the proposition, it suffices to produce one sextic surface $S$ such that $F(S)_{0}$ is a smooth irreducible curve.

Take a general quartic threefold (in short q.t.) $Y \subseteq \mathbf{P}^{4}$ with two nodes, say $U, V$. Let $F(Y)=$ Fano variety of conics contained in $Y$, $F(Y)_{U}$ (resp. $\left.F(Y)_{V}\right)$, be the closed subset of conics passing through $U$ (resp. $V$ ) and smooth in that point (cf. [10]). Then, applying the proof of [10, Lemma 1] to the linear system $\mathscr{Y}(U, V)$ of q.t. which are singular in $U$, and $V$, we get, for general $Y$, that $F(Y)$ is a reduced surface. Moreover, $F(Y)_{V}$ is a smooth connected curve (hence irreducible). To see this, consider the linear system $\mathscr{Y}(V)$ of q.t. singular in $V$. In $\mathscr{Y}(V)$ there is a divisor whose general element correspond to a q.t. with exactly two nodes as its only singularities. If $Z \subset \mathscr{Y}(V)$ is the subset of q.t. $Y$ such that $F(Y)_{V}$ is singular, then it follows from [10 Lemma 3] that $\operatorname{codim}(Z) \geq 2$. So, we can find a point $V$ and $Y \in \mathscr{Y}(V)$ such that $F(Y)_{V}$ is smooth and the only singularities of $Y$ are the nodes $U, V$. By loc. cit. Lemma 4, and Zariski connectedness Theorem, $F(Y)_{V}$ is connected for all $Y \in \mathscr{Y}(V)$.

Now, fix homogeneous coordinates $\left(x_{0}: x_{1}: x_{2}: t: x_{3}\right)$ on $\mathbf{P}^{4}$ so that $U=(0: 0: 0: 1: 0), V(0: 0: 0: 0: 1)$ and $Y$ has equation 
Let $\sigma=$ blowing-up of $\mathbf{P}^{4}$ in $U, \pi=$ projection of $\mathbf{P}^{4}$ from $U$ onto $\mathbf{P}^{3}=\{t=0\}$. If $\tilde{Y}$ is the strict transform of $Y$ by $\sigma$, then $\pi \mid Y$ extends to a 2:1 map $\tilde{\pi}: \tilde{Y} \rightarrow \mathbf{P}^{3}$ branched over the sextic surface $S=$ discriminant locus of $(+)$. Since $Y$ is general in $\mathscr{Y}(U, V)$, the only singularities of $S$ are a node in $0=\pi(V)=(0: 0: 0: 1)$ plus the 24 points defined by $\left\{G_{2}=G_{3}\right.$ $\left.=G_{4}=0\right\}$. We define now an embedding of $\overline{F(Y)}$ in $F(S) \subseteq \mathscr{Z}_{2}$. Let

$W=$ variety of conics of $\mathbf{P}^{4}$ which are smooth in $U$ and let $w$ : $\tilde{W} \rightarrow W$ be the blowing-up of $W$ along the subvariety $W^{1}$ of conics passing through $U$. As for the case of $\tilde{\mathscr{Z}}_{2}$ studied in (2.7), one can show that $\tilde{W}$ parametrizes the following family of curves in $\tilde{\mathbf{P}}^{4}$ :

$$
\left\{\sigma^{-1}(C) \text {, for } C \in W-W^{1}\right\} \cup\left\{\tilde{C}+L, \text { for } C \in W^{1}\right\}=M \cup N
$$

where $\tilde{C}=$ strict transform of $C$ by $\sigma$ and $L \subseteq \sigma^{-1}(U)$ is a line through $\tilde{C} \cap \sigma^{-1}(U)$. Notice that all the curves of the above family are locally complete intersections in $\tilde{\mathbf{P}}^{4}$.

Now, the map $\pi$ induces a morphism $p: \tilde{W} \rightarrow \mathscr{Z}_{2}$ such that:

(a) if $m \in M$ parametrizes $\sigma^{-1}(C)$, then $p(m)=\pi_{*}(C)$;

(b) if $n \in N$ corresponds to $\tilde{C}+L$, then $p(n)=$ point parametrizing the singular curve $\pi(C)+L^{\prime}$, where $L^{\prime}=\pi(h), h=2$-plane containing the lines parametrized by $L$.

Notice that $\pi(C) \cap L^{\prime}=\pi$ (line tangent to $C$ )

Let $\overline{F(Y)}$ be the strict transform of $F(Y)$ in $\tilde{W}$. We claim that

$$
p \mid \overline{F(Y)}=p^{\prime}: \quad \overline{F(Y)} \rightarrow \mathscr{Z}_{2}
$$

is a smooth injective morphism; so $p^{\prime}$ embeds $\overline{F(Y)}$ in $F(S)$. An elementary computation of parameter shows that a general $Y$ neither contains two conics having the same image under $\pi$, nor a plane section through $U$ and $V$ which is a conic counted twice. This implies that $p^{\prime}$ is injective. To see that $p^{\prime}$ is smooth, consider $f \in \overline{F(Y)}$. Then, either $f \in M$ or $f \in N$. Using the notation introduced in (a) and (b), let $H$ denote either the hyperplane containing $C$ and $U$, or the one containing $U, \pi(C)$ and $L^{\prime}$. Let $\tilde{H}=$ strict transform of $H$ in $\tilde{P}^{4}, \tilde{H}_{2}=$ strict transform in $\tilde{W}$ of the variety of conics contained in $H$. Since the fibre $p^{-1}(p(f))$ is contained in $\tilde{H}_{2}$, to show that $p^{\prime}$ is smooth we need to show that $T_{\tilde{H}_{2}, f} \cap T_{\widetilde{F(Y)}, f}=(0)$. This is equivalent to saying that the component of $T_{\widetilde{F(Y)}, f}$ along the direction $\tilde{H}_{2}$ is $(0)$. This component is clearly identified with $H^{0}\left(f, N_{f / K}\right)$, where $K=H \cdot Y$ and $f=$ curve corresponding to $f$, and since $K$ is a $K 3$ surface, it is 0 . Notice that $p^{\prime}(\overline{F(Y)})=F^{\prime}=F(S)$ (lines counted twice). Also, one easily verifies that $F^{\prime}=p^{\prime}(F(Y)) \cup \mathbf{P}^{3}$, where $\mathbf{P}^{3}=$ projective space of all conics totally tangent to $S$ which are obtained intersecting each plane in $\mathbf{P}^{3}$ with the tangent cone $\left\{G_{2}=0\right\}$ to $Y$ in $U$. 
Consider now a general family $\left\{S_{z}, z \in \Delta=\right.$ unit disc $\}$ of sextic surfaces which are singular in 0 , and such that $S_{0}=S$. By Proposition 1.10 , we can assume $\operatorname{dim}\left(F\left(S_{z}\right)\right)=2$ for all $z \neq 0$. In $\mathscr{Z}_{2}^{\prime}=\mathscr{Z}_{2}-\{$ lines counted twice $\}$, consider the Zariski closure $\bar{F}$ of the surface $U_{0 \leq|z| \leq 1}$ $F\left(S_{z}\right)_{0}-\mathbf{P}^{2}$, where $\mathbf{P}^{2} \subset \mathbf{P}^{3}$ is the subfamily of conics parametrized by the above $\mathbf{P}^{3}$ and passing through 0 .

The fibre over 0 of the natural morphism $q: \bar{F} \rightarrow \Delta$ is the curve $F(Y)_{0}$, which is smooth and irreducible. So, the same must hold true for the general fibre of $q$.

Proposition 2.19. Let $S$ be a general sextic with a node, then:

(i) $\overline{F(S)}-\tilde{I}$ is smooth, where $\tilde{I}=\{\tilde{Q} \in \overline{F(S)}: n(\tilde{Q})$ is a branch point for $\left.n_{0}\right\}$;

(ii) if $Q \in F(S)_{0}$ and $n^{-1}(Q)=\left\{Q_{1}, Q_{2}\right\}$ with $Q_{1}$ and $Q_{2}$ distinct, then $T_{1} \cap T_{2}=T_{F(S)_{0}, Q}$, where $T_{i}=\operatorname{dn}\left(T_{\widetilde{F(S)}, Q_{1}}\right)$, $i=1$,2. In particular $\operatorname{dim}\left(T_{1} \cap T_{2}\right)=1$.

Proof. As in the proof of Proposition (2.17), set $Q=\left(Q^{\prime}, Q^{\prime \prime}\right), F(S)$ $=F$, etc. Clearly, to prove the proposition, it suffices to show that $\tilde{F}$ is smooth along $\tilde{F}_{0}-\tilde{I}$. Assume $Q \in F_{0}, Q=$ origin in $\mathbf{A}^{30} \times \mathbf{A}^{8}$. Recall that locally

$$
\widetilde{F(S)} \subseteq\left(\mathbf{A}^{30} \times \mathbf{A}^{3}\right) \times\left(\mathbf{A}^{8} \times \mathbf{A}^{1}\right)=\left(\ldots ; \lambda_{0}, \lambda_{1}, \lambda_{2}\right) \times(\ldots ; \lambda)
$$

and that $n$ is the restriction to $\tilde{F}$ of the linear projection onto $\left\{\lambda=\lambda_{0}=\right.$ $\left.\lambda_{1}=\lambda_{2}=0\right\}$. Hence, as a linear morphism of affine tangent spaces, $d n_{Q_{i}} \mid T_{\tilde{F}, Q_{i}}{ }^{i}=1,2$, is just the same projection. Also, since $Q_{1} \neq Q_{2}$, these two restriction maps are injective (see Proposition 2.17). Now, by (2.14), $\tilde{F}$ is contained in $\left\{u_{3}-\lambda t_{3}=0\right\}$; so it follows that in $\mathbf{A}^{30} \times \mathbf{A}^{3}, T_{1} \subseteq\left\{u_{3}\right.$ $\left.-\lambda^{\prime} t_{3}=0\right\}, T_{2} \subseteq\left\{u_{3}-\lambda^{\prime \prime} t_{3}=0\right\}$, where $\lambda^{\prime}$ (resp. $\lambda^{\prime \prime}$ ) is the $\lambda$-coordinate of $Q_{1}$ (resp. $Q_{2}$ ). On the other hand, it is clear from $\S 1$ that

$$
T_{1} \cup T_{2} \subseteq T_{F, Q}=(0) \times T_{\mathscr{S}, Q^{\prime \prime}} \subseteq T_{\mathscr{D}, Q} \subseteq T_{\mathscr{Z}_{2}, Q^{\prime}} \times T_{\mathscr{Z}_{6}, Q^{\prime \prime}}
$$

Moreover, $T_{F, Q} \subseteq T_{\mathscr{D}, Q}$ is identified with the linear subsystem of (+) $T_{\mathscr{D}, Q}=\{$ linear system $(1.6)+(1.7)\}$ given by (in the affine coordinates introduced in (2.3))

$(++) g_{2} g_{4}+g_{3}^{2}+h_{5}\left(t_{1} x+t_{2} y+t_{3}\right)=0, \quad z=t_{1} x+t_{2} y+t_{3}$.

Thus, $T_{1}$ (resp. $T_{2}$ ) is the intersection of $(++)$ with the linear subsystem $M_{1}$ (resp. $M_{2}$ ) of $(+)$, obtained substituting $u_{3}$ with $\lambda^{\prime} t_{3}$ (resp. $\lambda^{\prime \prime} t_{3}$ ). Clearly, $\operatorname{dim} T_{i}=2, i=1,2$, iff the above intersections are proper iff

$$
V=M_{1} \cap T_{F, Q} \cap\left\{t_{3}=0\right\}=M_{2} \cap T_{F, Q} \cap\left\{t_{3}=0\right\}
$$


is 1-dimensional. Since locally in $\tilde{F}, \tilde{F}_{0}=\left\{t_{3}=0\right\}$, it follows that $V=$ $(d n)_{Q_{1}}\left(T_{\tilde{F}_{0}, Q_{1}}\right)=(d n)_{Q_{2}}\left(T_{\tilde{F}_{0}, Q_{2}}\right)$. By Proposition $2.17, Q$ is a smooth point for $F_{0}$; since $n_{0}$ is unramified in $Q, Q_{1}$ and $Q_{2}$ are smooth for $\tilde{F}_{0}$ and $\operatorname{dim}(V)=1$.

As in Proposition 2.19 set

$$
I=\left\{\text { branch points of } n_{0}\right\}, \quad \tilde{I}=n^{-1}(I) .
$$

We show that $\overline{F(S)_{0}}$ has ordinary double points in $\tilde{I}$, that $\overline{F(S)}$ is not normal along $\tilde{I}$ and that each $\tilde{Q} \in \tilde{I}$ is a double point for $\widetilde{F(S)}$. In particular, the normalization of $\widehat{F(S)}$ in $\tilde{Q}$ is smooth and $\tilde{Q}$ has two distinct preimages.

To see this consider the double covering

$$
f: X \rightarrow \mathbf{P}^{3}
$$

branched over $S . X$ has an ordinary double point in $V=f^{-1}(0)$ and no other singularities. If $C$ is a curve parametrized by a point $c \in F(S)$ then $f^{*}(C)$ splits; i.e.,

$$
f^{*}(C)=C^{\prime}+C^{\prime \prime}
$$

where $\operatorname{deg}\left(f / C^{\prime}\right)=\operatorname{deg}\left(f / C^{\prime \prime}\right)=1$ and $C^{\prime}, C^{\prime \prime}$ are connected. This defines a double covering

$$
g: F(X) \rightarrow F(S)
$$

where

$$
F(X)=\left\{C^{\prime}, C^{\prime \prime} \text { as in } 2.21\right\}
$$

and $g$ sends the points corresponding to the curves $C^{\prime}, C^{\prime \prime}$ to the point $c$. Since no conics lie in $S g$ is unramified.

Then consider the cartesian square

$$
\begin{array}{ccc}
\tilde{X} & \stackrel{\tilde{\boldsymbol{\sigma}}}{\rightarrow} & X \\
\tilde{f} \downarrow & & \downarrow f \\
\tilde{\mathbf{P}}^{3} & \rightarrow & \mathbf{P}^{3}
\end{array}
$$

where $\sigma$ blows up $\mathbf{P}^{3}$ in $0, \tilde{\sigma}$ blows up $X$ in $V$ and desingularizes it. If

$$
T=\tilde{f}^{-1}\left(\sigma^{-1}(0)\right)=\text { exceptional divisor of } \tilde{\sigma}
$$

then $T$ is a smooth quadric surface. Moreover $\tilde{f} / T: T \rightarrow \mathbf{P}^{2}=\sigma^{-1}(0)$ is branched over the smooth conic

$$
B=\tilde{S} \cap \sigma^{-1}(0),
$$


( $\tilde{S}=$ strict transform of $S$ ). Notice also that the lines of the two rulings on $T$ are the preimages by $\tilde{f}$ of the lines tangent to $B$.

Take now a curve $D$ corresponding to a point $\tilde{Q}$ of $\overline{F(S)} ; f^{*}(D)$ splits again in two copies of $D$. We want to point out what happens when $\tilde{Q} \in \widetilde{F(S)_{0}}$. In this case $D=\tilde{C}+L$, (with $\tilde{C}=$ strict transform of a t.t. conic smooth in $0, L=$ line tangent to $B, \tilde{C}+L$ connected). Since $f^{*}(C)$ splits in two copies $C^{\prime}, C^{\prime \prime}$ of $C$ we have

$$
f^{*}(\tilde{C}+L)=\tilde{C}^{\prime}+\tilde{C}^{\prime \prime}+L_{1}+L_{2}
$$

where $\tilde{C}^{\prime}, \tilde{C}^{\prime \prime}$ are the strict transforms of $C^{\prime}, C^{\prime \prime}, \tilde{f}^{*}(L)=L_{1}+L_{2}$ and $L_{1}, L_{2}$ are the lines of $T$ through $\tilde{f}^{-1}(L \cap B)$. Also:

$$
C^{\prime} \cap C^{\prime \prime}=\varnothing \quad \text { iff } C \cap B=\varnothing \quad \text { iff } \tilde{Q} \notin \tilde{I} .
$$

In this case there exist exactly 2 (connected) copies of $\tilde{C}+L$ which can be obtained from 2.25. On the other hand, if $C \cap B=\{P\}$, then $C^{\prime} \cap$ $C^{\prime \prime} \cap L_{1} \cap L_{2}$ contains a unique point $\tilde{P}=f^{-1}(P)$ and there exist four copies of $\tilde{C}+L$ in $f^{*}(\tilde{C}+L)$. Set now

$$
\begin{aligned}
& \widetilde{F(X)}=\{\text { curves } \tilde{D} \subseteq \tilde{X}: \tilde{D} \text { is connected, } \\
& \qquad \tilde{f}(\tilde{D}) \in \widetilde{F(S)}, \operatorname{deg}(\tilde{f} / \tilde{D})=1\},
\end{aligned}
$$

there is a commutative diagram

$$
\widetilde{F(X)} \stackrel{\tilde{n}}{\rightarrow} F(X)
$$

$$
\begin{array}{ccc}
\tilde{g} \downarrow & & \downarrow g \\
F(S) & \stackrel{n}{\rightarrow} & F(S)
\end{array}
$$

where $\tilde{n}$ sends $\tilde{D}$ in $\tilde{\sigma}(\tilde{D})$ and $\tilde{g}$ sends $\tilde{D}$ in $\tilde{f}(\tilde{D})$. Consider also the commutative diagram of curves

$$
\begin{array}{ccc}
\tilde{g}^{-1}\left(\overline{F(S)_{0}}\right)=\widetilde{F(X)_{0}} & \stackrel{\tilde{n}_{0}}{\rightarrow} & F(X)_{0}=g^{-1}\left(F(S)_{0}\right) \\
\frac{\tilde{g}_{0} \downarrow}{\overline{F(S)_{0}}} & \overrightarrow{n_{0}} & \downarrow g_{0} \\
& & F(S)_{0}
\end{array}
$$

where the morphisms are the restrictions of the previous ones. $F(S)_{0}$ is smooth by Proposition 2.18, $g_{0}$ is finite; hence $g_{0}$ is flat and, being unramified, it is an etale double covering. In particular

$F(X)_{0}$ is a smooth curve.

Notice now that the cardinality of the fibre of $\tilde{n}_{0}$ is always 2; for, if $C^{\prime} \in F(X)_{0}$, then $\tilde{n}_{0}^{-1}\left(C^{\prime}\right)=\tilde{C}^{\prime}+L_{1}, \tilde{C}^{\prime}+L_{2}$ (with $\tilde{C}^{\prime}, L_{1}, L_{2}$ as in 
2.25). Hence, by the same argument as above

$$
\widetilde{F(X)_{0}} \text { is a smooth curve; }
$$

(in particular, from 2.27 and 2.30 , we have also that $\overline{F(X)}$ is of pure dimension). Now consider $\tilde{Q} \in \tilde{I}$, by the discussion after 2.25

$$
\# \tilde{g}_{0}^{-1}(\tilde{Q})=4 \text {. }
$$

Then, since $\operatorname{deg}\left(\tilde{g}_{0}\right)=2$ and $\widetilde{F(X)_{0}}$ is smooth, $\tilde{Q}$ is an ordinary double point for $\widetilde{F(S)_{0}}$ and

$$
\tilde{I}=\operatorname{Sing}\left(\widetilde{F(S)_{0}}\right)=\left\{\text { nodes of } \widetilde{F(S)_{0}}\right\} .
$$

Moreover $\overline{F(S)}$ is not normal along $\tilde{I}$ because $\operatorname{deg}(\tilde{g})=2$ and $\# g^{-1}(\tilde{Q})$ $=4, \forall \tilde{Q} \in \tilde{I}$. Consider the normalization diagram

$$
\begin{array}{ccccc} 
& & \widetilde{F(X)} & \stackrel{\tilde{n}}{\rightarrow} & F(X) \\
& g^{\nu} \swarrow & \frac{\downarrow \tilde{g}}{} & & \downarrow g \\
F(S)^{\nu} & \underset{\nu}{\rightarrow} & \widetilde{F(S)} & \underset{n}{\rightarrow} & F(S)
\end{array}
$$

where $\nu$ is the normalization morphism. Notice that $\nu$ is biregular on $\overline{F(S)}-\nu^{-1}(\tilde{I})$ and that, for all $\tilde{Q} \in \tilde{I}, \# \nu^{-1}(\tilde{Q})=2$. Moreover, $\tilde{n}_{0}$ is clearly the trivial double cover, and

$$
F(S)_{0}^{\nu} \text { is a smooth disconnected curve. }
$$

In addition observe that

$$
F(S)^{\nu} \text { is a smooth surface; }
$$

indeed, $F(S)_{0}^{\nu}$ is smooth along $\nu^{-1}(\tilde{I})$ and locally complete intersection on $F(S)^{\nu}$ (its equation is $\nu^{*} t_{3}=0$ ).

From the above discussion we deduce the following propositions

Proposition 2.35. Let $S$ be a general sextic surface with a node, then $F(X)_{0}$ is a smooth, irreducible curve.

Proof. From 2.11 we know that $F(X)_{0}$ is a smooth curve. Let us show that $F(X)_{0}$ is connected. Since $F(S)_{0}$ is irreducible $F(X)_{0}$ is not connected iff the double covering $g_{0}: F(X)_{0} \rightarrow F(S)_{0}$ is trivial. Denote by $Z \subseteq \tilde{\mathbf{P}}^{3}$ the strict transform by $\sigma$ of the union of all conics parametrized by $F(S)_{0}$. If $g_{0}$ is trivial $\tilde{f}^{*}(Z)=Z^{\prime}+Z^{\prime \prime}$, where $Z^{\prime}, Z^{\prime \prime}$ are two copies of $Z$ and $i_{*}\left(Z^{\prime}\right)=Z^{\prime \prime}$ ( $i=$ involution interchanging the sheets of $\tilde{f})$. 
Notice that, by [2], [9], $\operatorname{Pic}(\tilde{X})=\mathbf{Z} e \oplus \mathbf{Z} h,(e=$ class of $T, h=\tilde{h}-$ $e, \tilde{h}=$ pull-back of a plane by $(\tilde{f} \circ \sigma))$.

Take a general plane $H \subseteq \mathbf{P}^{3},(0 \notin H)$, and set $A=\tilde{f}^{-1}(\sigma(H)) . A$ is a smooth $K 3$ surface and $\tilde{f} / A: A \rightarrow H$ is a double covering of $H$ branched on $H \cap S$. It is easy to see that, for a general $S$ with a node, $H \cap S$ is a general sextic curve. Hence $\operatorname{Pic}(A)=\mathbf{Z}$ and, for every plane curve $C \subseteq H,(\tilde{f} / A)^{*}(C)$ does not split. This is a contradiction because $(\tilde{f} / A)^{*}(Z \cap H)$ splits.

Proposition 2.36. Let $S$ be a general sextic surface with a node, then

(i) every point $Q \in F(X)_{0}$ is a double point of rank 2 for $F(X)$;

(ii) assume $P \in F(X)_{0}$ and $\left\{P_{1}, P_{2}\right\}=\tilde{n}^{-1}(P)$, then

$$
T_{1} \cap T_{2}=T_{F(X)_{0}, P}
$$

where $T_{l}(i=1,2)$, is the image by dn of the tangent space to $F(X)$ in $P_{l}$. In particular $\operatorname{dim}\left(T_{1} \cap T_{2}\right)=1$.

Proof. By $2.34 F(S)^{\nu}$ is smooth and $g^{\nu}$ is unramified so that $\widetilde{F(X)}$ is smooth. $\overline{F(X)}$ is the fibre product of the morphisms $g, r=n \circ \nu$ and $r$ normalizes $F(S)$. Hence $\tilde{n}$ is the normalization morphism for $F(X)$. This implies that each $p \in F(X)$ is a double point and that the number of branches at $p$ is always 2 (the same holds for $F(S)$ ).

We want to see that $\operatorname{rk}\left(C_{p}\right)=2\left(C_{p}=\right.$ tangent cone in $p$ to $\left.F(X)\right)$. At first consider $\mathscr{Q} \in F(X)_{0}-g^{-1}(I)$ and set $\left\{\mathscr{Q}_{1}, \mathscr{Q}_{2}\right\}=\tilde{n}^{-1}(\mathscr{Q}),\left\{Q_{1}, Q_{2}\right\}$ $=n^{-1}(Q), \mathscr{T}_{i}=T_{\widetilde{F(X)}, \mathscr{Q}_{2}}, T_{i}=T_{F(S), Q_{i}}(i=1,2)$. Assume also that $\tilde{g}\left(\mathscr{Q}_{i}\right)$ $=Q_{i}$. By 2.32 the following diagram commutes:

$$
\begin{array}{ccc}
\mathscr{T}_{1} \oplus \mathscr{T}_{2} & \stackrel{\tilde{n}_{1}-\tilde{n}_{2}}{\rightarrow} & T_{F(X), \mathscr{Q}} \\
\tilde{g}_{1} \oplus \tilde{g}_{2} \downarrow & & \downarrow d g_{\mathscr{Q}} \\
T_{1} \oplus T_{2} & \stackrel{n_{1}-n_{2}}{\rightarrow} & T_{F(S), Q}
\end{array}
$$

where $\tilde{n}_{i}=d \tilde{n}_{\mathscr{Q}_{\ell}}, n_{i}=d n_{Q_{i}}, \tilde{g}_{l}=d \tilde{g}_{\mathscr{Q}_{i}}(i=1,2)$. Observe that $\tilde{g}$ is etale at $Q_{\imath}$ because $Q_{i}$ is smooth in $\widehat{F(S)}$ and $\# g^{-1}\left(Q_{i}\right)=2$. Hence $\tilde{g}_{i}$ is injective. By Proposition $2.19 \operatorname{dim}\left(\operatorname{Ker}\left(n_{1}-n_{2}\right)\right)=\operatorname{dim}\left(\operatorname{Im}\left(n_{1}\right) \cap\right.$ $\left.\operatorname{Im}\left(n_{2}\right)\right)=1$, so that, since $\tilde{g}_{1}-\tilde{g}_{2}$ is injective, $\operatorname{dim}\left(\operatorname{Im}\left(\tilde{n}_{1}\right) \cap \operatorname{Im}\left(\tilde{n}_{2}\right)\right) \leq 1$. On the other hand, since both $\overline{F(X)_{0}}$ and $F(X)_{0}$ are smooth, $\tilde{n}_{1}\left(T_{\widetilde{F(X)_{0}}, \mathscr{Q}_{1}}\right)=\tilde{n}_{2}\left(T_{\widetilde{F(X)_{0}}, \mathscr{Q}_{2}}\right)=T_{F(X)_{0}, \mathscr{2}}$. Hence $\operatorname{dim}\left(\operatorname{Im}\left(\tilde{n}_{1}\right) \cap \operatorname{Im}\left(\tilde{n}_{2}\right)\right)=$ 1. This shows (i) and (ii) for all $\mathscr{Q} \in F(X)_{0}-g^{-1}(I)$. In order to prove (i), (ii) for $\mathscr{Q} \in g^{-1}(I)$ use similar notation and also set $\tilde{T}=T_{\overline{F(S)}, \tilde{Q}}$. It 
suffices to show that $\tilde{n}_{1}-\tilde{n}_{2}$ is surjective. Consider from 2.34 the diagram

$\begin{array}{ccc}\mathscr{T}_{1} \oplus \mathscr{T}_{2} & \stackrel{\tilde{n}_{1}-\tilde{n}_{2}}{\rightarrow} & T_{2} \\ \tilde{g}_{1}-\tilde{g}_{2} \downarrow & & \downarrow d g_{9} \\ \tilde{T} & \stackrel{d n_{Q}}{\rightarrow} & T_{Q}\end{array}$

and observe that $\operatorname{Im}(d n)_{Q} \subseteq \operatorname{Im}(d g)_{2}$. Hence it suffices to show that $\tilde{g}_{1}-\tilde{g}_{2}$ is surjective. Since $d \tilde{g}=d \nu \circ d g^{\nu}$ this is equivalent to saying that $\operatorname{dim}\left(\operatorname{Im} \nu_{1} \cap \operatorname{Im} \nu_{2}\right)=1\left(\nu_{l}=d \nu_{Q_{l}^{\nu}},\left\{Q_{l}^{\nu}\right\}=\nu^{-1}(Q), i=1,2\right)$. To see this recall that $\widehat{F\left((S)_{0}\right.}$ has a node in $\tilde{Q}$ : let $W$ be the 2 dimensional vector space spanned by the images in $\tilde{T}$ if $T_{F(S)_{0}^{\nu}, Q_{1}^{\nu}}, T_{F(S)_{0}^{\nu}, Q_{2}^{\nu}}$. Since $n(\tilde{Q})$ is smooth for $F(S)_{0}$ we have $\operatorname{Ker}\left(d n_{\tilde{Q}}\right) \subseteq W$, (cf. Prop. 2.17). Moreover $\operatorname{Im} \nu_{i} \not \subset W$ because $n \circ \nu_{l}$ is injective $\left(\operatorname{Im}\left(n \circ \nu_{1}\right)=\operatorname{Im}\left(n \circ \nu_{2}\right)=\right.$ tangent cone in $Q$ to $F(S))$. This implies $\operatorname{dim}\left(\operatorname{Im} \nu_{1} \cap \operatorname{Im} \nu_{2}\right)=1$.

3. Let $f: X \rightarrow \mathbf{P}^{3}$ be a double covering of $\mathbf{P}^{3}$ branched along a sextic surface $S$. As in $\S 2$, set $F(X)=$ variety parametrizing connected curves $C \subseteq X$ such that

$$
f_{*}(C) \in F(S)
$$

and call $F(X)$ the Fano variety of conics contained in $X$. Notice that, if $X$ is smooth, then $C \cdot\left(-K_{x}\right)=2$ : for this reason we call $C$ a conic. There is a natural 2:1 morphism

$$
g: F(X) \rightarrow F(S)
$$

induced by $f$. If $S$ is smooth, $g$ is an étale covering except for points of $F(S)$ corresponding to conics contained in $S$.

As we mentioned in the introduction, the main result of this paper will be a consequence of the following proposition.

Proposition 3.2. Let $\left\{S_{t}\right\}\left(t \in \mathbf{P}^{1}\right)$ be a generic pencil of sextic surfaces, and let $\left\{X_{t}\right\}\left(t \in \mathbf{P}^{1}\right)$ be the corresponding family of double solids. Suppose $X_{t}$ is smooth except for $t=t_{1}, \ldots, t=t_{n}$. Then there exist $t_{n+1}, \ldots, t_{n+m} \in \mathbf{P}^{1}$ such that:

(i) for $t \in \mathbf{P}^{1}-\left\{t_{1}, \ldots, t_{n}, \ldots, t_{n+m}\right\}, F\left(X_{t}\right)$ is a smooth irreducible surface;

(ii) for $1 \leq i \leq m, F\left(X_{t_{n+1}}\right)$ has only isolated singularities;

(iii) for $1 \leq j \leq n, F\left(X_{t}\right)$ is an irreducible surface which is singular only along the curve $F\left(X_{t}\right)_{0}$ of conics passing smoothly through the ordinary double point of $X_{t_{j}} . F\left(X_{t_{j}}\right)_{0}$ is irreducible, and along this curve $F\left(X_{t_{1}}\right)$ is analytically reducible in two smooth components meeting transversally. 
Proof. We can assume, for all $t$, that $S_{t}$ does not contain any conic. The smoothness of $F\left(X_{t}\right)$ in (i) follows from (3.1) by Proposition 1.10. Applying to a general curve of $F(S)$ the argument used in the proof of Proposition 2.35, one easily gets the irreducibility of $F\left(X_{t}\right)$. To see (ii), recall from Proposition 1.10 that the variety $A$ parametrizing special sextics, is of codimension 3 in the incidence correspondence. Thus, the threefold swept out by the $F\left(S_{t}\right)\left(t \in \mathbf{P}^{1}\right)$ will intersect $A$ in at most a finite number of points. Finally, (iii) is a direct consequence of Propositions 2.35 and 2.36 .

THEOREM. 3.3. If $X$ is a generic sextic double solid, and $F(X)$ is the Fano surface of conics contained in $X$, then the Abel-Jacobi mapping a: $\mathrm{Alb}(F(X)) \rightarrow J(X)$ is an isomorphism.

Proof. By [10 Prop. 2], the only thing that is left to show is that the map $a$ is not constant on $F(X)$. For this, if $C \subseteq X$ is a conic represented by a smooth point of $F(X)$, we will show that the codifferential $\alpha^{\prime}$ of the Abel-Jacobi map $\alpha: F(X) \rightarrow J(X)$, is not the zero map.

By [11 pp. 24-27], we have a commutative diagram:

$$
\begin{array}{ccc}
H^{0}\left(X, N_{X / E} \otimes K_{X}\right) & \stackrel{R}{\rightarrow} & H^{1}\left(X, \Omega_{X}^{2}\right)=\Omega_{J(X), 0} \\
r \downarrow & & \downarrow \alpha^{\prime} \\
H^{0}\left(C, N_{C / E} \otimes K_{X}\right) & \overrightarrow{\beta_{C}} & H^{0}\left(C, N_{C / X}\right)^{*}=\Omega_{F(X), C}
\end{array}
$$

$(+)$

where $R$ is the residue homomorphism, $r$ the restriction map, and $\beta_{C}$ fits in the following exact sequence:

$$
\begin{aligned}
(++) \quad 0 & \rightarrow H^{1}\left(N_{C / X}\right)^{*} \rightarrow H^{0}\left(N_{C / E} \otimes K_{X}\right) \rightarrow H^{0}\left(N_{X / E} \otimes K_{X} \otimes \mathcal{O}_{C}\right) \\
& \rightarrow H^{0}\left(N_{C / X}\right)^{*} \rightarrow H^{1}\left(N_{C / E} \otimes K_{X}\right) \rightarrow H^{1}\left(N_{X / E} \otimes K_{X} \otimes \mathcal{O}_{C}\right) \rightarrow 0
\end{aligned}
$$

In our case, from $(+)$ we get:

$$
\begin{array}{ccc}
H^{0}\left(\mathbf{P}^{3}, \mathcal{O}_{\mathbf{P}^{3}}(5)\right) & \stackrel{R}{\rightarrow} & H^{2,1}(X) \\
r \downarrow & & \downarrow \alpha^{\prime} \\
H^{0}\left(C, \mathcal{O}_{C}(5)\right) & \vec{\beta}_{C} & H^{0}\left(C, N_{C / X}\right)^{*}
\end{array}
$$

and, since $N_{C / E}=\mathcal{O}_{C}(1) \oplus \mathcal{O}_{C}(2) \oplus \mathcal{O}_{C}(3)$, we obtain from $(++)$ the exact sequence

$$
\begin{aligned}
0 & \rightarrow H^{0}\left(\mathcal{O}_{C}(0) \oplus \mathcal{O}_{C}(1) \oplus \mathcal{O}_{C}(2)\right) \rightarrow H^{0}\left(\mathcal{O}_{C}(5)\right) \\
& \rightarrow H_{C}^{0}\left(N_{C / X}\right)^{*} \rightarrow 0
\end{aligned}
$$


Since $r$ is clearly onto, it follows that $\alpha^{\prime} \circ R$ is onto. From the isomorphism $H^{0}\left(\mathbf{P}^{3}, \mathcal{O}_{\mathbf{P}^{3}}(5)\right) / J \rightarrow H^{2,1}(X)$, where $J=$ jacobian ideal generated by the partial derivatives of the equation defining in $\mathbf{P}^{3}$ the branch locus $S$, it follows that $\alpha^{\prime}$ is onto.

\section{REFERENCES}

[1] W. Barth and A. Van de Ven, Fano varieties of lines on hypersurfaces, Arch. Math., 31 (1978), 96-104.

[2] C. H. Clemens, Double solids, Adv. Math., 47 (1983), 107-230.

[3] _ On the surjectivity of the Abel-Jacobi mapping, Ann. of Math.

[4] - Some results about Abel-Jacobi mappings, Transcendental topics in Algebraic Geometry, Annals of Math. Studies, 1983.

[5] _ Degeneration Techniques in the Study of Threefolds, in Algebraic Threefolds, Varenna 1981, Springer-Verlag 1982.

[6] H. Clemens and P. Griffiths, The intermediate Jacobian of the cubic threefold, Ann. of Math., 95 (1982), 281-356.

[7] A. Collino, The Abel-Jacobi isomorphism for the cubic fivefold, to appear in Pacific J. Math.

[8] P. Griffiths, Complex analysis and algebraic geometry, Bull. Amer. Math. Soc., 1 (1979), 595-626.

[9] V. A. Iskovskih, Fano threefolds I and II, Math USSR, 11 (no. 3) (1977), 485-527; 12 (no.3) (1978), 469-506.

[10] M. Letizia, The Abel-Jacobi mapping for the quartic threefold, Inven. Math., 75 (1984), 477-492.

[11] G. Welters, Abel-Jacobi isogenies for certain types of Fano threefolds, Mathematisch Centrum Amsterdam, 1981.

Received November 30,1984. Both authors are partially supported by a grant from the Italian Ministry of Public Education and the C.N.R. of Italy.

UNIVERSITA DI TORINO

via Principe Amedeo, 8

10123 TORINO, ITALY 



\section{PACIFIC JOURNAL OF MATHEMATICS EDITORS}

\author{
V. S. VARADARAJAN \\ (Managing Editor) \\ University of California \\ Los Angeles, CA 90024 \\ HeRbert Clemens \\ University of Utah \\ Salt Lake City, UT 84112 \\ R. FINN \\ Stanford University \\ Stanford, CA 94305
}

HERMANN FLASCHKA

University of Arizona

Tucson, AZ 85721

RAMESH A. GANGOLLI

University of Washington

Seattle, WA 98195

VAUGHAN F. R. JONES

University of California

Berkeley, CA 94720

ROBION KIRBY

University of California

Berkeley, CA 94720
C. C. MOORE

University of California

Berkeley, CA 94720

H. SAMELSON

Stanford University

Stanford, CA 94305

HAROLD STARK

University of California, San Diego

La Jolla, CA 92093

\section{ASSOCIATE EDITORS}
R. Arens
E. F. BECKENBACH
B. H. NEUMANN
F. WOLF
K. YoshIDA (1906-1982)

\section{SUPPORTING INSTITUTIONS}

UNIVERSITY OF ARIZONA

UNIVERSITY OF BRITISH COLUMBIA

CALIFORNIA INSTITUTE OF TECHNOLOGY

UNIVERSITY OF CALIFORNIA

MONTANA STATE UNIVERSITY

UNIVERSITY OF NEVADA, RENO

NEW MEXICO STATE UNIVERSITY

OREGON STATE UNIVERSITY
UNIVERSITY OF OREGON

UNIVERSITY OF SOUTHERN CALIFORNIA

TANFORD UNIVERSITY

UNIVERSITY OF HAWAII

UNIVERSITY OF TOKYO

UNIVERSITY OF UTAH

WASHINGTON STATE UNIVERSITY

UNIVERSITY OF WASHINGTON 


\section{Pacific Journal of Mathematics}

Vol. 124, No. $1 \quad$ May, 1986

Kinetsu Abe and Martin Andrew Magid, Relative nullity foliations and indefinite isometric immersions

Erik P. van den Ban, A convexity theorem for semisimple symmetric

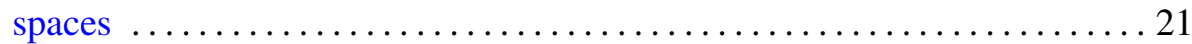

Bo Berndtsson and Thomas Joseph Ransford, Analytic multifunctions, the $\bar{\partial}$-equation, and a proof of the corona theorem .................. 57

Brian Boe and David H. Collingwood, Intertwining operators between

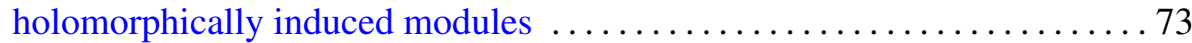

Giuseppe Ceresa and Alessandro Verra, The Abel-Jacobi isomorphism for

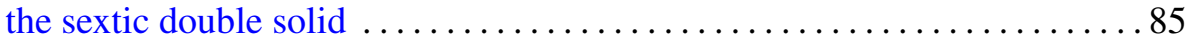

Kun Soo Chang, Jae Moon Ahn and Joo Sup Chang, An evaluation of the conditional Yeh-Wiener integral ........................... 107

Charles Dale Frohman, Minimal surfaces and Heegaard splittings of the

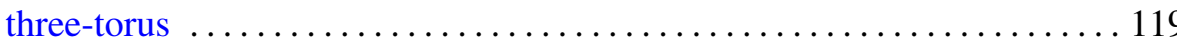

Robert M. Guralnick, Power cancellation of modules $\ldots \ldots \ldots \ldots \ldots \ldots 131$

Kenneth Hardy and Kenneth S. Williams, On the solvability of the Diophantine equation $d V^{2}-2 e V W-d W^{2}=1 \ldots \ldots \ldots \ldots \ldots \ldots \ldots \ldots \ldots$

Ray Alden Kunze and Stephen Scheinberg, Alternative algebras having

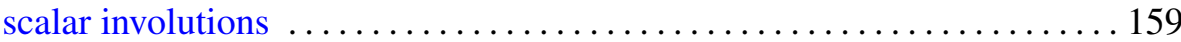

W. B. Raymond Lickorish and Kenneth Millett, The reversing result for the Jones polynomial $\ldots \ldots \ldots \ldots \ldots \ldots \ldots \ldots \ldots$

Guido Lupacciolu, A theorem on holomorphic extension of CR-functions

William Schumacher Massey and Lorenzo Traldi, On a conjecture of K. Murasugi

Dinakar Ramakrishnan, Spectral decomposition of $L^{2}(N \backslash \mathrm{GL}(2), \eta)$

Steven L. Sperber, On solutions of differential equations which satisfy certain algebraic relations 